\title{
Stroke in a Young Adult Secondary to Vitamin B12 Deficiency induced Hyperhomocysteinemia
}

\author{
${ }^{1}$ LS Yashaswini, ${ }^{2}$ Mamatha B Patil
}

\begin{abstract}
Stroke in young adults is less frequent than in older population but has major impact on individual and society. Stroke in young justifies an extensive etiologic work up-both common and uncommon. Pernicious anemia is characterized by lack of intrinsic factor in gastric mucosa which is essential for absorption of vitamin B12. Deficiency of vitamin B12 contributes to hyperhomocysteinemia which promotes both arterial and venous thrombosis. We present a rare case of stroke in young adult caused by hyperhomocysteinemia secondary to vitamin B12 deficiency.
\end{abstract}

Keywords: Homocysteine, Pernicious anemia, Stroke, Vitamin B12.

How to cite this article: Yashaswini LS, Patil MB. Stroke in a Young Adult Secondary to Vitamin B12 Deficiency induced Hyperhomocysteinemia. J Med Sci 2015;1(1):10-13.

\section{Source of support: Nil}

Conflict of interest: None

\section{INTRODUCTION}

Stroke in young adults occurs in individuals less than 45 years of age and accounts for $10 \%$ of all strokes. ${ }^{8}$ In India, nearly one-fifth of patients with first ever strokes admitted to hospital are aged less than 40 years. It justifies an extensive etiologic work up as conventional risk factors, such as hypertension, hyperlipidemia, and smoking do not account for all these cases. Risk factors for stroke in young are cardioembolic (35\%), large vessel atherosclerosis $(12 \%)$, lacunar infarcts $(7 \%)$, collagen vascular diseases ( $3 \%)$, and indeterminate origin $(43 \%) .{ }^{1}$ In India, cerebral venous thrombosis and rheumatic heart disease are the leading causes of stroke followed by tubercular meningitis. ${ }^{2}$ Stroke caused by hyperhomocysteinemia secondary to vitamin B12 deficiency is a very rare and preventable cause of ischemic stroke.

\footnotetext{
${ }^{1}$ Assistant Professor, ${ }^{2}$ Professor

1,2Department of Medicine, RajaRajeswari Medical College \& Hospital, Bengaluru, Karnataka, India
}

Corresponding Author: Mamatha B Patil, Professor Department of Medicine, RajaRajeswari Medical College \& Hospital, Kambipura, Mysore Road, Bengaluru, Karnataka India, Phone: 9845680586, e-mail: id-dr.mamatharamesh@ yahoo.in

\section{CASE REPORT}

Here, we present a case report of a 34-year-old male patient who was admitted to emergency room of our hospital on 16-7-2013, with complaints of weakness of right upper limb and lower limb of 2 days duration which he developed suddenly while driving an autorickshaw. Patient did not give any history of loss of consciousness, vomiting, seizures. He was not a known case of hypertension, diabetes mellitus, ischemic heart disease. Patient did not give history of substance abuse. Patient gave history of headache and blurring of vision in right eye 2 years back which resolved completely after 5 days.

On examination, patient was moderately built and nourished with height of $170 \mathrm{~cm}$ weighing $60 \mathrm{Kg}$. His pulse was 84 beats/minute, blood pressure $140 / 90 \mathrm{~mm} \mathrm{Hg}$, he did not have pallor. On central nervous system examination, patient was conscious and oriented. Optic fundus showed grade 2 hypertensive retinopathy, upper motor neuron (UMN) facial palsy was present on right side. On motor system examination, there was hypertonia with power of $3 / 5$ in right upper and lower limb. Plantar reflex was extensor on right side. Sensory and cerebellar system examination was normal. Other systems' examination was also normal.

On investigating, patient hemoglobin was $13.6 \mathrm{gm} \%$, mean corpuscular volume (MCV) was $100 \mathrm{fl}$, and erythrocyte sedimentation rate (ESR) was $80 \mathrm{~mm}$ at the end of one hour, peripheral smear showed macrocytosis. Total leucocyte and platelet counts were normal. Random blood glucose was $100 \mathrm{mg} / \mathrm{dl}$, blood urea nitrogen (BUN) $-13 \mathrm{mg} / \mathrm{dl}$, serum creatinine $-0.7 \mathrm{mg} / \mathrm{dl}$ and serum electrolytes were normal. Fasting lipid profile showed cholesterol $-72 \mathrm{mg} / \mathrm{dl}$, high density lipoprotein (HDL) - 27, low density lipoprotein (LDL) - 39, very low density lipoprotein (VLDL) - 12, triglycerides-61 mg/dl. Venereal disease research laboratory (VDRL), HbsAg, HIV were negative. aPTT-22s (C-27s), prothrombin time13s (C-11s), international normalized ratio (INR)-1.15. As peripheral smear examination showed macrocytosis, we got serum vitamin B12 levels estimated which was low $(159 \mathrm{pg} / \mathrm{ml}$, normal range-174-878 pg/ml). Serum homocysteine was $>50 \mathrm{micromol} / 1$ (normal-4.9-15 micromol/1). Serum folic acid levels were normal. Computed tomography (CT) scan of brain (Fig. 1) showed 


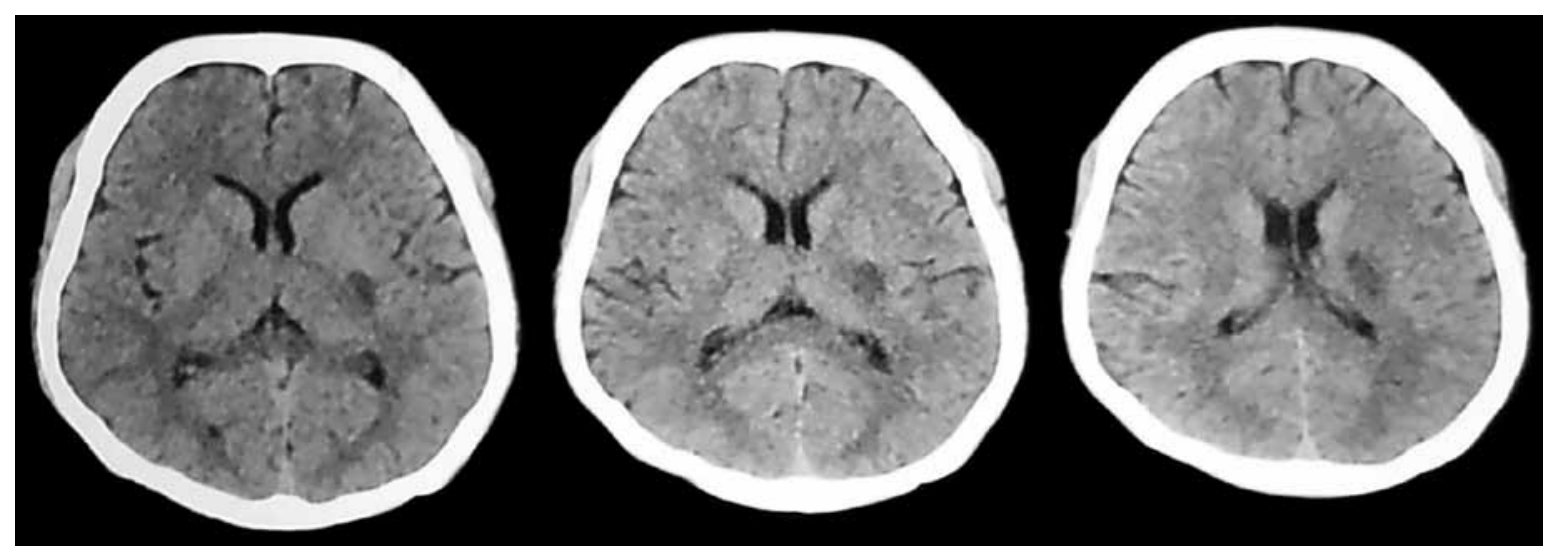

Fig. 1: Computed tomography of brain

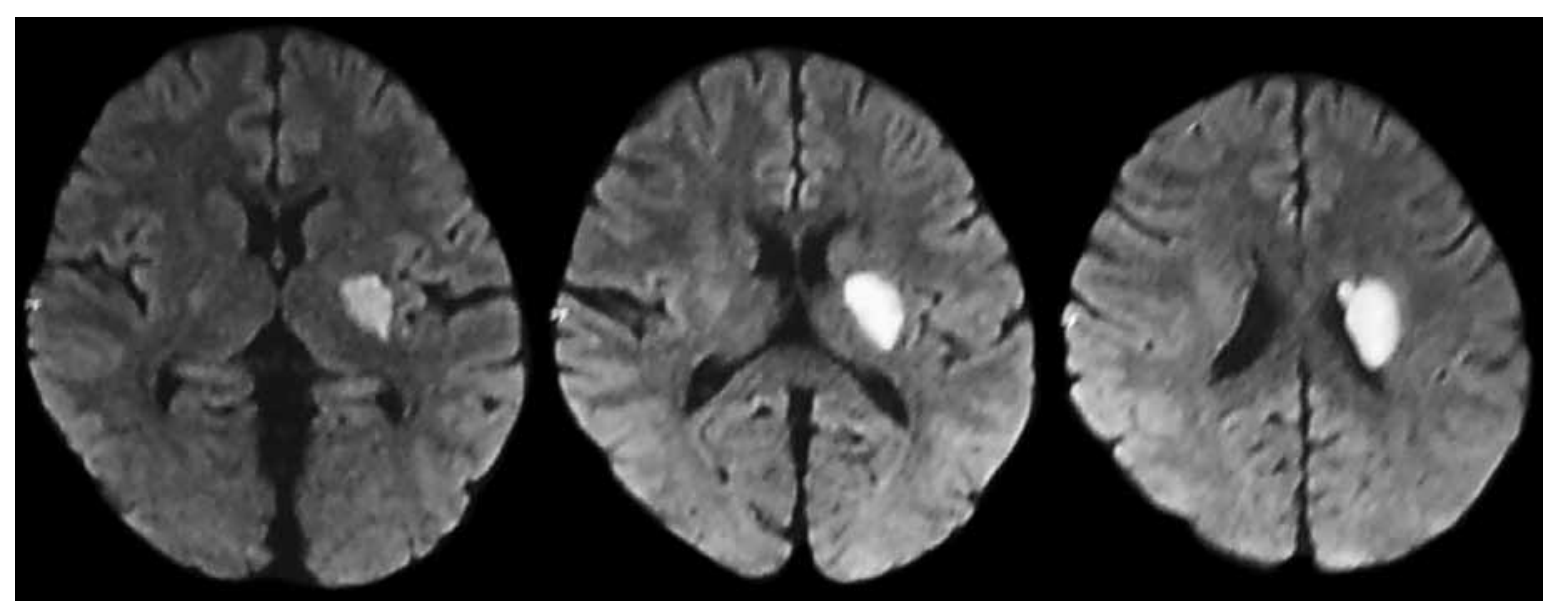

Fig. 2: Magnetic resonance imaging of brain

cerebral infarct in posterior limb of internal capsule, lentiform nucleus, periventricular white matter, posterior aspect of corona radiata on left side. Magnetic resonance imaging (MRI) of brain (Fig. 2) showed infarcts in the same areas mentioned in CT scan, i.e. in left middle cerebral artery territory, MR angiogram was normal. Carotid Doppler (Fig. 3) study showed fibrofatty plaque in posterior wall of right carotid bulb with no stenosis. Electrocardiogram, two-dimensional (2D) echocardiography and ultrasound study of abdomen were normal. Upper GI endoscopy showed mucosal atrophy which was suspicious of pernicious anemia and subsequently gastric biopsy was done which showed nonspecific gastritis. Serum antiparietal and anti-intrinsic factor antibodies were negative.

\section{COURSE IN THE HOSPITAL}

Patient was treated with cyanocobalamin, folic acid and pyridoxine injections and subsequently patient's power in right upper and lower limb improved to $4 / 5$.

\section{DISCUSSION}

Vitamin B12 is a water soluble vitamin with high stores in liver (2-10 $\mathrm{mg}$ ) and deficiency of this vitamin is as such rare. Deficiency is more common in pure vegans than nonvegetarians as animal foods, such as meat, eggs, fish and milk, are rich sources of this vitamin.? The recommended daily allowance (RDA) is $2.4 \mathrm{mg} /$ day in adults and $2.6 \mathrm{mg} /$ day in pregnant and lactating women. ${ }^{7}$ Causes of vitamin B12 deficiency are varied like inadequate intake, pernicious anemia, malabsorption syndromes, etc.

Pernicious anemia was first described by Thomas Addison and anemia associated with it is called Biermer disease. ${ }^{7}$ It is characterized by lack of intrinsic factor in gastric mucosa which is essential for absorption of vitamin B12 in ileum. It is an autoimmune disease with antibodies directed against parietal cells or intrinsic factor. However, antibodies in serum can be absent in around $10 \%$ of these patients.

\section{METABOLISM OF HOMOCYSTEINE}

Deficiency of vitamin B12 and/or folic acid in serum inhibits conversion of homocysteine to methionine, increasing levels of homocysteine in serum. Thus, plasma vitamin B12 levels and homocysteine levels are inversely related. Low vitamin B12 concentration contributed to $28 \%$ of hyperhomocysteinemia, while low 

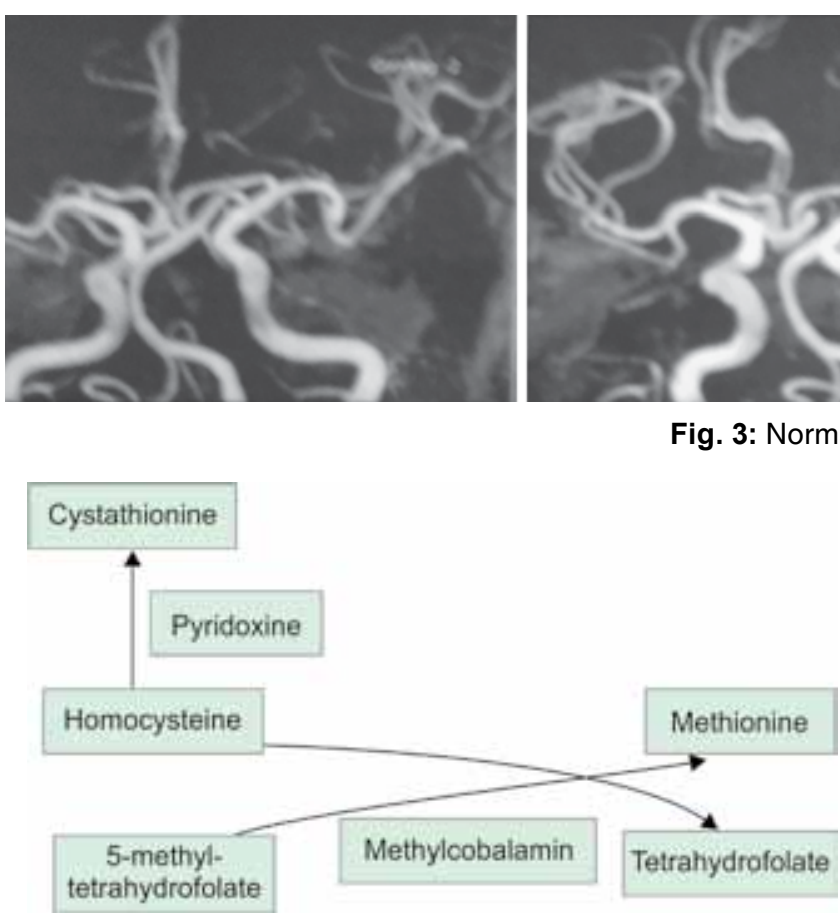

Fig. 4: Normal metabolism of homocysteine

folate concentration to $2 \%$ of hyperhomocysteinemia. ${ }^{5}$ Homocysteine is a highly reactive amino acid and is toxic to vascular endothelium, potentiates auto oxidation of LDL and promotes both arterial and venous thrombosis. Hence, these patients with hyperhomocysteinemia secondary to vitamin B12 deficiency are at high risk for coronary heart disease, cerebrovascular disease and peripheral vascular disease. ${ }^{3}$

Deficiency of vitamin B12 causes both hematological and neurological manifestations. Hematological manifestations are megaloblastic anemia, pancytopenia, hypersegmented neutrophils. Macrocytosis may be absent when folate concentration is high, and serum ferritin levels are low. ${ }^{5}$ Neurological manifestations include the following:

- Central nervous system-dementia, depression, acute psychosis, cerebrovascular accident.

- Spinal cord-myelopathy.

- Peripheral nervous system-subacute combined degeneration, peripheral neuropathy. ${ }^{7}$

Hyperhomocysteinemia is associated with few components of metabolic syndrome, like hyperglycemia, hypertension and low HDL cholesterol..$^{5}$ This might explain fundoscopic changes of hypertensive retinopathy and low lipid levels in our patient. Hyperhomocysteinemia is a risk factor for recurrent stroke which is possibly the cause for symptoms of blurring of vision and headache in our patient 2 years back.

In a similar case report, a 45 -year-old woman sustained two ischemic cerebral infarctions 16 years after ileal resection for Crohn's disease in the absence of anemia. Her evaluation showed an elevated random serum homocysteine level, a low serum vitamin B12 level, and an increased mean corpuscular volume (MCV) without anemia. A methionine-loading test resulted in a marked increase in the homocysteine levels 2, 4, and 6 hours after the load. A Schilling test demonstrated a malabsorption of vitamin B12. Vitamin B12 injections normalized her fasting homocysteine level and her MCV. She had no recurrent strokes during a year of follow-up. ${ }^{4}$

Even though, low circulating vitamin B12 concentration has been recognized in Indians, there has been little appraisal of this. Serum vitamin B12 and homocysteine are not routinely measured in clinical practice as most patients are asymptomatic. ${ }^{5}$

Supplementation of vitamin B12, folic acid to these patients would reduce homocysteine levels in blood which in turn gives protection against recurrent stroke. ${ }^{6}$

\section{CONCLUSION}

Stroke in young patients needs a thorough investigation looking into broad array of potential etiologies-both common and uncommon. Stroke caused by hyperhomocysteinemia secondary to vitamin B12 deficiency is a very rare and preventable cause of ischemic stroke. Thus, stroke in young requires a different approach in both investigations and management than stroke in the elderly. We as healthcare providers must stress on prevention of stroke, especially by identifying treatable risk factors both genetic and acquired. These preventive measures could aid immensely in bringing down costs and emotional burden on the family.

\section{REFERENCES}

1. Kristensen B, et al. Epidemiology and etiology of ischemic stroke in young adults aged 18 to 44 years in Northern Sweden. Stroke 1997;28(9):1702-1709.

2. Tripathi M, Vibha D. Stroke in young in India. Stroke Res Treat 2011;2011:368629. 
3. Clarke R, et al. Hyperhomocysteinemia: an Independent risk factor for vascular disease. N Engl J Med 1991 Apr 25;324(17): 1149-1155.

4. LaRoy P. Penix, ischemic stroke secondary to vitamin B12 deficiency induced hyperhomocysteinemia. Neurology 1998 Aug;51(2):622-624.

5. Yagnik CS, et al. Vitamin B12 deficiency and hyperhomocysteinemia in rural and urban Indians. JAPI 2006;54:775-782.
6. Bougteba A, Basir A, Kissani N. Recurrent ischemic stroke revealing Biermer's disease. Rev Neurol (Paris) 2009 Dec; 165(12):1099-1102.

7. Sethi N, Robilotti E, Sadan Y. Neurological manifestations of vitamin B12 deficiency. The Int J Nutrition and Wellness 2005;2(1). DOI: 10.5580

8. Griffiths D, Sturm J. Epidemiology and etiology of young stroke. Stroke Research and Treatment 2011;2011:209370. 